\title{
Engineering
}

\section{Electromyography-informed modeling for estimating muscle activation and force alterations in Parkinson's disease}

Marco Romanato, Daniele Volpe, Annamaria Guiotto, Fabiola Spolaor, Massimo Sartori \& Zimi Sawacha

To cite this article: Marco Romanato, Daniele Volpe, Annamaria Guiotto, Fabiola Spolaor, Massimo Sartori \& Zimi Sawacha (2021): Electromyography-informed modeling for estimating muscle activation and force alterations in Parkinson's disease, Computer Methods in Biomechanics and Biomedical Engineering, DOI: 10.1080/10255842.2021.1925887

To link to this article: https://doi.org/10.1080/10255842.2021.1925887

Published online: 17 May 2021.

Submit your article to this journal $๘$

Lll Article views: 116

Q View related articles $\sqsubset$

View Crossmark data $[\pi$ 


\title{
Electromyography-informed modeling for estimating muscle activation and force alterations in Parkinson's disease
}

\author{
Marco Romanato ${ }^{a}$, Daniele Volpe ${ }^{b}$, Annamaria Guiotto $^{a}$, Fabiola Spolaor ${ }^{a}$, Massimo Sartori ${ }^{c}$ and \\ Zimi Sawacha ${ }^{\mathrm{a}, \mathrm{d}}$ \\ ${ }^{a}$ Department of Information Engineering, University of Padua, Padova, Italy; ${ }^{b}$ Fresco Parkinson Center, Villa Margherita, Vicenza, Italy; \\ 'Department of Biomechanical Engineering, University of Twente, AE Enschede, Netherlands; ${ }^{\mathrm{d}}$ Department of Medicine, University of \\ Padua, Padova, Italy
}

\begin{abstract}
Electromyography (EMG)-driven neuromusculoskeletal modeling (NMSM) enables simulating the mechanical function of multiple muscle-tendon units as controlled by nervous system in the generation of complex movements. In the context of clinical assessment this may enable understanding biomechanical factor contributing to gait disorders such as one induced by Parkinson's disease (PD). In spite of the challenges in the development of patient-specific models, this preliminary study aimed at establishing a feasible and noninvasive experimental and modeling pipeline to be adopted in clinics to detect PD-induced gait alterations. Four different NMSM have been implemented for three healthy controls using CEINMS, an OpenSim-compatible toolbox. Models differed in the EMG-normalization methods used for calibration purposes (i.e. walking trial normalization and maximum voluntary contraction normalization) and in the set of experimental EMGs used for the musculotendon-unit mapping (i.e. 4 channels vs. 15 channels). Model accuracy assessment showed no statistically significant differences between the more complete model (non-clinically viable) and the proposed reduced one (clinically viable). The clinically viable reduced model was systematically applied on a dataset including ten PD's and thirteen healthy controls. Results showed significant differences in the neuromuscular control strategy of the PD group in term of muscle forces and joint torques. Indeed, PD patients displayed a significantly lower magnitude on force production and revealed a higher amount of force variability with the respect of the healthy controls. The estimated variables could become a measurable biomechanical outcome to assess and track both disease progression and its impact on gait in PD subjects.
\end{abstract}

\section{ARTICLE HISTORY}

Received 3 September 2020

Accepted 1 May 2021

\section{KEYWORDS}

Parkinson's disease; neuromusculoskeletal modeling; EMG-informed approach; gait analysis; neuromuscular disorders; rehabilitation

\section{Introduction}

Parkinson's disease (PD) is the second most prevalent neurodegenerative disorder, affecting people worldwide (de Lau and Breteler 2006). In the upcoming decades PD will is expected to increase and develop to the as a Parkinson pandemic-scale, doubling the number of affected patients by 2040 (Dorsey and Bloem 2018). PD is a neurodegenerative pathological condition characterized by a dopamine deficiency causing a cohort of motor and non-motor symptoms including tremor, rigidity, and bradykinesia. The middle-advanced phase can manifest axial disorders which includes postural deformities, postural instability, and gait disorders (Tiple et al. 2009). For a motoric point of view, gait alterations are among the most relevant hallmarks of this neurodegenerative disorder; i.e. abnormal increment of cadence, stance and double support phases have been interpreted as a compensatory strategy to the reduction of both stride length and lower limb joints range of motion (Morris et al. 2005). Recently, the authors detected a further marker of motor disorder in PD patients, by identifying specific alterations in both timing (Spolaor et al. 2017) and amplitude (Volpe et al. 2020) of leg muscle electromyograms (EMGs) during the gait cycle in comparison with matched healthy subjects. Advances is PD treatment have been hampered by a lack of an objective and reliable biomarkers that can describe the disease onset, progress, and modulation in response to therapies (Marek et al. 2011). Established assessment to PD-induced motor dysfunctions relies on a qualitative assessment. That is, the subjective observation of an individual physician delivering clinical scales, e.g. the Movement Disorder Society - Unified PD Rating Scale 
(Movement Disorder Society Task Force on Rating Scales for Parkinson's Disease 2003), which may highly differ from physician to physician, thereby yielding different assessment for the same patient. In some cases, these are coupled with instrumented gait analysis (Morris et al. 2005) and recently a several approaches based on machine learning techniques have been developed for pathological gait classification. These methods have proven their reliability either in discriminating between PD subjects and healthy controls (Daliri 2013; Rastegari et al. 2019), or in disease diagnosis and motor impairment characterization (Shetty \& Rao 2016; Caramia et al. 2018; Ricciardi et al. 2019). However, current motion capture techniques do not provide insights on the interplay between neural activation to muscles and disrupted musculoskeletal function. Although these are both established tools for clinical practice, measuring causal information about neural-to-mechanical function in the PD patients in vivo, is not possible. As an alternative, personalized data-driven computer modeling and simulation techniques can be used to provide a quantitative objective assessment metric for patients' motor function: combining gait analysis and a forward dynamics modeling approach, i.e. EMGinformed modeling (Sartori et al. 2016, 2017), could provide a deeper understanding of the biomechanical mechanism underlying disease progression. This methodology has been successfully applied across a range of neuromuscular pathologies (Scarton et al. 2017) and healthy individuals (Varotto et al. 2017). In this context a subject specific neuromusculoskeletal model (NMSM) is created for each individual, driven by their own neural-excitation patterns provided by the EMG signals. In comparison with inverse dynamics approaches (Veerkamp et al. 2019), which rely on cost functions tailored on healthy humans' motion and do not consider pathological neural strategies, the proposed data-driven simulation allows either capturing the patient's true muscle activation patterns and identifying patient-specific neuromuscular parameters, thus serving the basis for the definition of quantitative and reliable biomarkers; estimation of muscle force would be the most direct variable explain changes in the subject's gait function, overall strength as well as disease progression over time. Moreover, it could be used to identify the target of different rehabilitation programs, to predict outcomes across different therapies and the effect of different modulations within the treatment.

Currently, no studies investigated the possibility to quantitatively assess key biomechanical factors such as strength in terms of muscle forces as a biomarker of disease progression in PD subjects (Skinner et al. 2015); besides this, little is known on how a change in co-activation and co-contraction would affect muscle force and torques around a specific joint in PD differently from healthy subjects. Furthermore, despite the potential of adopting NMSM, these models are rarely introduced in clinical practice mainly due to the difficulty of validating the results of muscle forces, while the experimental setup and acquisition protocol might result complex and long. Indeed, the main limitations when assessing patients in neurological conditions occurs in obtaining realistic maxima voluntary contractions (MVC) for model calibration purposes and in the number of required experimental EMG signals, which might be cumbersome for patients.

The aim of this work is to address the extraction of information about PD neuromuscular functions that could not be measurable in vivo. Thus, the adoption of a clinically viable experimental and modeling pipeline for the estimation of muscle force, may enables to lay the foundations for the generation of new biomechanical-based biomarkers by computing reliable muscle-tendon forces together with multi-muscle activation strategies, which directly relates to changes in gait control and strength over time in PD. The aim of this work is to address the extraction of information about PD neuromuscular functions that could not be measurable in vivo, thus laying the foundations for the generation of new biomarkers by computing reliable muscle-tendon forces together with muscle activations strategy, which directly relates to changes in strength over time in PD throughout the adoption of a NMSM purposely adapted for the clinical practice. Additionally, a co-contraction ratio index (CCR) is computed to describe the simultaneous activity of the agonist-antagonist muscles spanning the knee and ankle joints (Busse et al. 2005). The impact of the proposed experimental and modeling pipeline simplification Hence, we was evaluated in a sample of healthy subjects the impact of a minimally invasive model, against models of enhanced complexity already existing pipelines, which result too lengthy for patients on neurological conditions in a sample of healthy subjects. Even though we do not expect to reach the same precision of a standard NMSM (Sartori et al. 2015), we hypostasize that the NMSM driven with the reduced set of muscles could still be informative for treatment planning in PD. Indeed, when evaluating PD patients, an EMG-driven modeling pipeline is needed as the commonly used 
inverse dynamics techniques for muscle force estimation (e.g. Static Optimization) would yield activation results deviating substantially from the patient's EMGs.

\section{Methods}

\section{Participants}

Two different datasets were involved in the study following the approval by the local Ethic Committees (CE/PROG 61/16-19/11/2015 (Peppe et al. 2019); University Policlinic of Padova protocol $\mathrm{n}^{\circ} 1001 \mathrm{P}-$ 21/11/2005 (Sawacha et al. 2010)). The first dataset (Dataset 1) included ten PD patients (6 males, 4 females, age $=66.89 \pm 12.78$ years, $\mathrm{BMI}=$ $23.38 \pm 3.35 \mathrm{~kg} / \mathrm{m}^{2}$ ), and 13 control subjects matched for age and BMI (CS, 7 males, 6 females, age = $58.23 \pm 11.48$ years, BMI $\left.=25.63 \pm 3.34 \mathrm{~kg} / \mathrm{m}^{2}\right)$. The PD participants had a Hoen and Yahr scale of 2-3 (Hoen \& Yahr 1967) and Mini Mental State Evaluation $>24$ (Folstein et al. 1975). Moreover, patients were able to walk autonomously and to perform the required tasks, on a stable treatment regimen for at least 3 months with a disease duration $>$ 5 years and had a good response to anti-Parkinsonian therapy. The measures have been done at the same time in the morning in off medication in order to avoid the effect of L-Dopa as documented by some authors (Morris et al. 2005). PD patients with Deep Brain Stimulation were excluded from the study.

The second dataset (Dataset 2) composed by three healthy subjects (age $=60 \pm 1$ years, BMI $=$ $27.4 \pm 4.7 \mathrm{~kg} / \mathrm{m}^{2}$ ) was retrospectively selected from the database available at the Human Movement Bioengineering Laboratory for model sensitivity assessment purposes, according to the following inclusion criteria: healthy subjects with no neurological conditions and orthopedic diseases; matched for age and BMI with Dataset 1; raw EMG data available and MVC acquisition for the following muscles, according to (Sartori et al. 2015): Gluteus Maximus, Gluteus Medius, Tensor Fasciae Latae, Adductor Longus, Sartorius, Semitendinosus, Biceps Femoris (BF), Rectus Femoris (RF), Vastus Medialis, Vastus Lateralis, Peroneus Longus, Gastrocnemius Medialis, Gastrocnemius Lateralis (GL), Soleus and Tibialis Anterior (TA).

\section{Data collection}

Both datasets have been acquired at the Human Movement Bioengineering Laboratory (10 m walkway) of the Department of Information Engineering at the University of Padova (Italy) equipped with 6 cameras stereophotogrammetric system $(60-120 \mathrm{~Hz}$, BTS S.r.l., Italy), synchronized with two force plates $(960 \mathrm{~Hz}$, Bertec Corporation, USA). A modified version of the IOR-Gait protocol (Del Din et al. 2011) was adopted for anatomical landmarks identification. Data collection included static acquisitions for anatomical calibration (Cappozzo et al. 1995) as well as several self-selected speed gait trials. For EMG recording, two protocols were adopted on Dataset 1 and Dataset 2 respectively:

Dataset 1: the EMG signal of 4 muscles (RF, BF, GL, TA) was bilaterally recorded by means of an 8channel EMG system $(1000 \mathrm{~Hz}$, BTS Free, BTS S.r.l., Italy).

Dataset 2: the EMG signal of 15 muscles (Gluteus Maximus, Gluteus Medius, Tensor Fasciae Latae, Adductor Longus, Sartorius, Semitendinosus, BF, RF, Vastus Medialis, Vastus Lateralis, Peroneus Longus, Gastrocnemius Medialis, GL, Soleus and TA) was unilaterally recorded, through a 16-channel EMG system $(1000 \mathrm{~Hz}$, BTS Free, BTS S.r.l., Italy). Each muscle MVC was acquired as in (Mantoan et al. 2015) for model-calibration purposes.

Surface EMG electrodes were placed following the minimal crosstalk area guidelines to maximize the measured selective activity per muscle (Blanc and Dimanico 2010).

\section{Data processing and neuromusculoskeletal modeling}

Four NMSM of different complexity and normalization method were developed:

- an MVC based NMSM adopting the full protocol (MVCTNf);

- an MVC based NMSM adopting the reduced protocol (MVCTNr);

- an NMSM calibrated on maximum value of each muscle chosen from all the walking trials (WTN), as in Varotto et al. (2017), adopting the full protocol (WTNf);

- an NMSM calibrated on WTN adopting the reduced protocol (WTNr);

All the four NMSM were generated for Dataset 2, while for the Dataset 1 only the WTNr model was implemented, as a reduced EMG protocol was adopted and no MVC were acquired for this dataset. 
Table 1. Mapped musculotendon units.

\begin{tabular}{llll}
\hline Experimental muscle EMG full & Experimental muscle EMG reduced & Musculotendon unit & Knee/ankle ago-antagonist muscle \\
\hline RF & $\mathrm{RF}$ & rectfem & Knee extensor \\
Vastus Medialis & $\mathrm{RF}$ & vasmed & Knee extensor \\
Vastus Lateralis & $\mathrm{RF}$ & vaslat & Knee extensor \\
(Vastus Medialis + Vastus Lateralis)/2 & $\mathrm{RF}$ & vasmed & Knee extensor \\
$\mathrm{BF}$ & $\mathrm{BF}$ & bifemlh & Knee flexor \\
$\mathrm{BF}$ & $\mathrm{BF}$ & bifemsh & Knee flexor \\
Semitendinosus & $\mathrm{BF}$ & semiten & Knee flexor \\
Semitendinosus & $\mathrm{BF}$ & semimem & Knee flexor \\
GL & $\mathrm{GL}$ & latgas & Knee flexor/Plantar flexor \\
Gastrocnemius Medialis & $\mathrm{GL}$ & medgas & Knee flexor/Plantar flexor \\
Soleus & $\mathrm{GL}$ & sol & Plantar flexor \\
TA & $\mathrm{TA}$ & tibant & Dorsi flexor \\
\hline
\end{tabular}

Recorded EMG and their associated musculotendon units informed by these EMG. The units spanning a specific joint were gathered into agonist and antagonist groups based on the moment arm convention in (Sartori et al. 2012)

\section{Model generation}

Three left and three right foot strikes were considered for the processing when the foot was naturally landing on the force plates. MOtoNMS (Mantoan et al. 2015) was adopted to export the motion data used in OpenSim framework and to normalize the EMG. A generic musculoskeletal model (gait2392 (Delp et al. 1990)) was used to linearly scale each subjects' geometry in OpenSim, matching the virtual markers of the model with the experimental ones of the subjects acquired during the static pose trial. Inverse kinematics, inverse dynamics (ID) and muscle analysis tools were used to obtain joint angles and moments and musculotendon moments and moment arms during the recorded trials.

CEINMS (Pizzolato et al. 2015) was adopted as toolbox to estimate the muscle forces that best matched the experimental EMGs and joint moments. Two different methods were adopted to normalize the EMG signals for calibration: the WTN and the MVCTN. In the WTN, the peak amplitude of each EMG linear envelope was computed across all the subject's trials. The MVCTN method consists instead in calculating the maximum EMG value from the MVC acquisition. The implementation of the full setup reflected the one described in (Sartori et al. 2015), while in the reduced setup, 4 EMG channels were mapped to a total of 12 musculotendon units (Table 1) (Romanato et al. 2019).

Experimental EMGs were used to directly drive their associated muscles in CEINMS. Two degrees of freedom (ankle plantar-dorsi flexion and knee flexionextension) were analyzed separately. For each subject, all the dynamic trials were used both for the calibration and the execution. Calibration identifies subjectspecific parameters that vary nonlinearly across subjects' anthropometric features and force generating capacities, i.e. muscle twitch activation/deactivation time constants, EMG-to-activation nonlinearity factor, muscle optimal fiber length, tendon slack length, and muscle maximal isometric force (Durandau et al. 2019). After calibration, CEINMS was used to predict the knee and ankle moments, muscle forces and activations with a hybrid EMG-informed model, that combined both the forward dynamics and the static optimization procedure that minimally adjusts experimental EMG-excitations to minimize instantaneous EMG-predicted joint moment estimates; the initial nominal parameters are repeatedly refined as part of a least-squares optimization procedure so that the mismatch between the EMG-driven model's predicted joint moments and those measured experimentally is minimized (Sartori et al. 2014). The weighting factors of the hybrid function were obtained through an experimental analysis which identified the following best weighting values: the factor that multiplies the difference between the predicted moment and the experimental moment was set as $\alpha=1$; the factor that multiplies the square of the minimally adjusted excitation (MAE) for the MTU spanning the assessed joint was set as $\beta=7.5$; the difference between MAE and the experimental EMG-excitation for the MTU spanning the assessed joint was set as $\gamma=12$. In order to calculate the CCR, the formula in (Sartori et al. 2015) was implemented, were an index of -1 indicates a solely contribution of the agonist excitations, 1 indicates a contribution derived just from antagonist excitations and 0 indicates a balanced contribution of both the agonist and antagonist excitations, i.e. the maximum value of co-contraction.

\section{Models' sensitivity assessment}

In order to test the reliability of the $\mathrm{WTNr}$ model, the loss of information derived both from the low number of EMG signals experimentally acquired and 


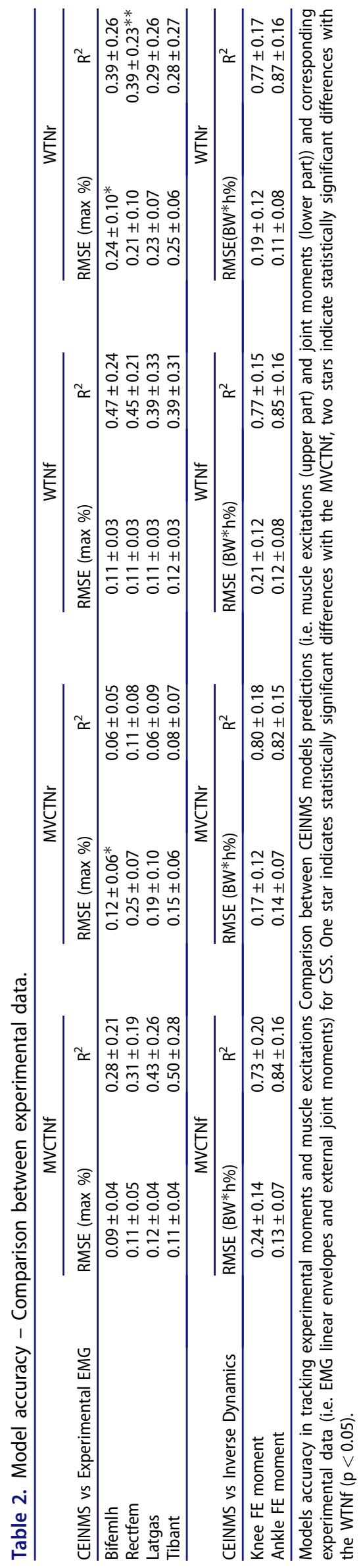

from the EMG envelope normalization across the maximum value of the dynamic trials was estimated.

Models were executed to predict muscle activations, joint torques and muscle forces in the four conditions and compared. All estimates were time normalized to the gait cycle duration and averaged across six walking trials. Root mean square error (RMSE) and coefficient of determination $\left(\mathrm{R}^{2}\right)$ between experimental and predicted muscle excitations and joint moments were computed to assess the physiological and biomechanical validity of each NMSM. Computed muscle forces were qualitatively compared via time-series plots. Oneway repeated measures ANOVA test with Bonferroni correction was used to determine whether the difference in the four implemented models was associated with differences in muscle forces time-series, with statistical significance $\mathrm{p}<0.05$.

\section{Statistical analysis}

In order to compare results of the PD subjects with the CS, the assessed variables were represented by the mean from six representative walking trials per subject, selected after applying an intra-class correlation threshold of 0.75 as in Del Din et al. (2011). The estimated variables (i.e. joint angles and torques, muscle forces and CCR) were plotted in percentage of the gait cycle and the 2 tailed Student's Ttest was used, after evidence of normality.

\section{Results}

In the following section results regarding the sensitivity of the NMSM to the different configurations and the comparison between the WTNr model applied to PD subjects and CS.

\section{Models' accuracy assessment}

All models accurately reproduced both the experimental joint moments (Table 2). $\mathrm{R}^{2}$ values were always greater than 0.7 across all trials and joints. Regarding muscle excitations, both full models produced small values of RMSE (RMSE $<0.11$ ). Although the WTNr produced the highest RMSE values (RMSE $<0.25$ ), it produced $\mathrm{R}^{2}$ coefficients comparable with the full models. Concerning muscle forces (Figure 1) statistically significant differences between the MVCTNf and WTNr were only detected in some phases of the gait cycle over the knee flexors. Overall, the WTN models produced forces of a lower magnitude with respect of the MVCTN models, with the only exception of the two gastrocnemii. 

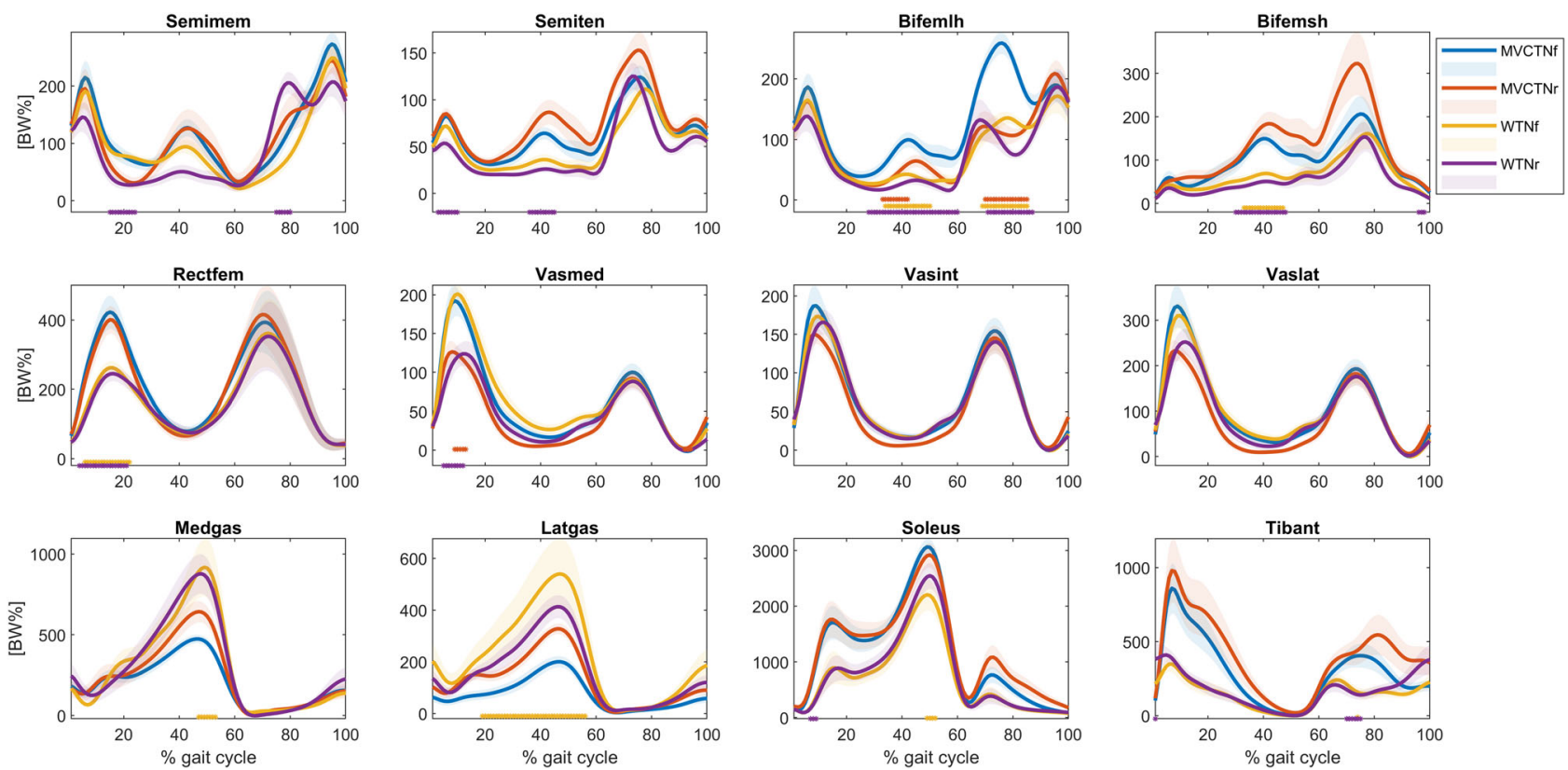

Figure 1. Muscle forces estimated with CEINMS for four different models. MVCTNf (in blue, \pm 1 standard error in shaded blue area), MVCTNr (in red, \pm 1 standard error in shaded red area), WTNf (in yellow, \pm 1 standard error in shaded yelow area), WTNr (in purple, \pm 1 standard error in shaded purple area). The muscle forces 0.05 .
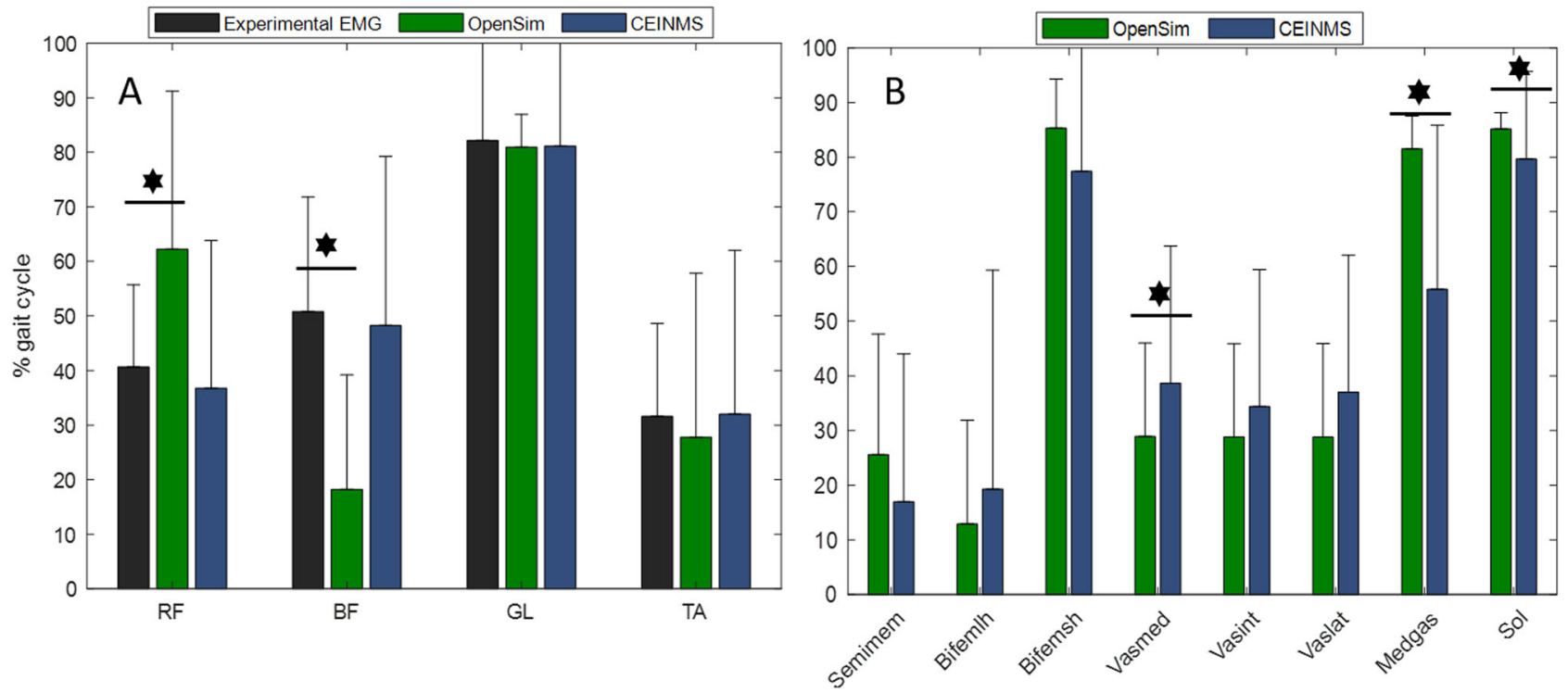

Figure 2. (A) Activation peaks position in percentage of the gait cycle for PD participants. Comparison between the experimental EMG activations, the activations directly driven from these EMGs in CEINMS, and their correspondent static optimization-derived activations in OpenSim. Experimentally measured muscle activation (in black), OpenSim derived ones for the same muscles (in green) and CEINMS derived ones for the same muscles (in blue). $\mathrm{RF}=$ rectus femoris, $\mathrm{BF}=$ bicept femoris, $\mathrm{LG}=$ gastrocnemius lateralis, $\mathrm{TA}=$ tibialis anterior. $* \mathrm{p}<0.05$. (B) Activation peaks position in percentage of the gait cycle for PD participants. Comparison between simulated muscles. EMG-informed activations (in blue) derived by the associated EMG (see Table 1), and OpenSim derived ones for the same muscles (in green). Semimem $=$ semimembranosus, Bifemlh=biceps femoris long head, Bifemsh = biceps femoris short head, Vas Med=vastus medialis, Vas Int=vastus intermedius, Vas Lat=vastus lateralis, Med Gas $=$ gastrocnemius medialis, Soleus $=$ soleus. $^{*} p<0.05$.

\section{WTNr model reliability in PD}

In order to verify the reliability of the WTNr model across the pathological population, the PD group envelopes peak percentage position within the gait cycle of the experimentally measured muscles and the model-derived ones (Figure 2A) were compared, as in Scarton et al. (2017) and in Hicks et al. (2015), as well as the PD ID-generated joint moments in OpenSim versus the CEINMS-estimated torques 
(Figure 3), as in Davico et al. (2020). In Figure 3 were also reported the joint kinematics of the sagittal plane obtained in OpenSim through the solution of an IK problem.

We observed statistically significant differences between the activations estimated with the static optimization approach and the experimental ones for RF $(\mathrm{p}<0.0001)$ and the BF $(\mathrm{p}<0.0001)$; no statistically significant differences were reported between the simulated activations calculated with the EMGinformed model and the experimentally measured ones (Figure 2A). Moreover, MTUs informed by the experimental EMGs presented statistically significant differences with respect to the one simulated with the static optimization based approach in terms of activations of vastus medialis $(\mathrm{p}<0.0239)$, gastrocnemius medialis $(\mathrm{p}<0.0001)$ and soleus $(\mathrm{p}<0.0232)$ (Figure 2B). Furthermore, the calibrated neuromusculoskeletal model proved to be dynamically consistent with the data provided by the ID OpenSim tool. Indeed, with respect of the ankle and knee flexion-extension moments, no statistically significant differences were found between ID and CEINMS-derived torques, with the only exception for the final phase of the stance.

\section{WTNr model outcomes}

With respect to sagittal plane joint kinematics obtained with the IK tool from OpenSim (Figure 4A), statistically significant differences were shown in PD group both in ankle plantar-dorsiflexion early-to-mid stance phase $(0.0023<\mathrm{p}<0.0499)$ and in knee flexion extension either in load acceptance $(0.0168<\mathrm{p}<$ $0.0481)$ and in mid-to-final stance phase $(0.0001<\mathrm{p}$ $<0.3974$ ). When considering joint moments (Figure $4 B$ ), statistically significant differences were appreciated between the two cohort of subjects, both in hill strike and push-off phases either for ankle $(0.0001<$ $\mathrm{p}<0.0163)$ or knee joints $(0.0001<\mathrm{p}<0.0461)$. CCR (Figure 4C) did not show statistically significant differences between the CS and the PD, but a greater variability in the second half of the stance phase for the knee joint was noticed (maximum standard deviation of 1.3503 in correspondence of the $62 \%$ of the stance phase). This suggests a lower neuromuscular control involvement at the knee joint level in that phase with respect to the more distal joint, and the adoption of different motor control strategies within the PD group. Although the CCR did not show statistically significant differences, the CC occurrence during five stages of the stance phase has been identified as the equal contribution furnished both by flexors and extensors muscles, i.e. $\mathrm{CCR}=0$; the results reported in Figure 4D highlighted different motor strategies in the PD group, especially for the ankle joint, where most of the CCs occurred after the load acceptance phase, where $50 \%$ of the CCs produced by the PD group were detected against the $28,57 \%$ produced by the CS.

Concerning muscle forces magnitude (Figure 5), statistically significant differences were shown between the two populations, except for the ankle joint plantar-flexor muscles during the stance phase. Major differences were highlighted in the BF long head $(0.0001<\mathrm{p}<0.0112)$, BF short head $(0.0001<$ $\mathrm{p}<0.0468)$, semitendinosus $(0.0001<\mathrm{p}<0.0485)$, semimembranosus $(\mathrm{p}<0.0001)$ and in the second half of the gait cycle of the RF $(p<0.0001$ between the $25 \%$ and $95 \%$ of the gait cycle). The vastii showed similar patterns during the stance phase in both the samples.

\section{Discussion}

The current study showed the possibility to capture patient's true muscle activation profile to assess reliable muscle forces for a neurological population of subjects through an EMG-driven NMSM informed with a reduced set of muscles and a simplified calibration method (WTNr). The accuracy of this modeling approach was tested considering all possible sources for loss of accuracy in estimating muscle activations for the simulated muscles. Largest RMSE were detected on BF activation profile in the MVCTNr (RMSE $=0.25 \pm 0.07)$ and in the knee flexion-extension moment in the MVCTNf (RMSE = $\left.0.24 \pm 0.14\left[\mathrm{BW}^{*} \mathrm{~h} \%\right]\right)$.

When considering the application of the WTNr model in the pathologic population, our results in terms of kinematics revealed in PD subjects a more dorsiflexed pattern during the stance phase of gait compared to the CS. The knee joint denoted a marked flexed pattern and a reduced joint angular excursion, as highlighted in literature (Lewis et al. 2000; Morris et al. 2005; Pistacchi et al. 2017; Volpe et al. 2017). Alterations in kinetics were found to be as marked as in kinematics. The moment produced at the loading response in the ankle joint was significantly reduced in PD group. As suggested in a previous work (Sofuwa et al. 2005), this might be due to a flat-footed gait pattern caused by a limitation in the hip-flexion, an inadequate knee extension and a partial heel strike which produce a ground reaction force vector closer to the ankle joint. Conversely with other 

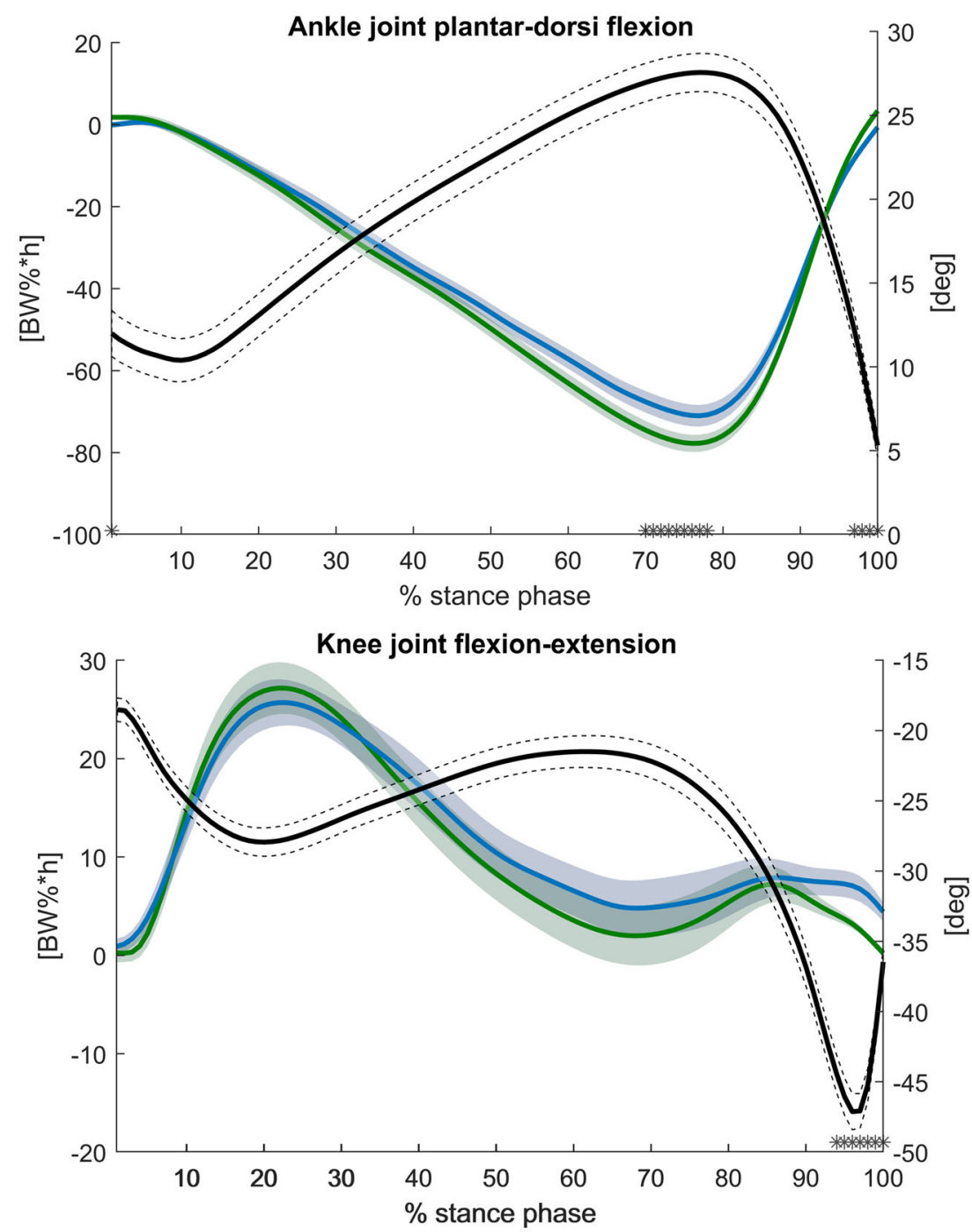

Figure 3. Joint angles in PD in percentage of the stance phase IK-generated angles and expressed in degrees (mean in black, \pm 1 standard error in shaded white area, right $y$-axes), and joint moments in PD subjects in percentage of the stance phase and normalized with respect to body weight $\times$ height $(B W \times h) \%$, IDgenerated moments from OpenSim (mean in green, \pm 1 standard error in shaded green area, left y-axes), CEINMS-estimated torques (mean in blue, \pm 1 standard error in shaded blue area, left $y$-axes). Top of the figure presents the ankle joint angles and moments, bottom of the figure knee joint angles and moments. $* \mathrm{p}<0.05$ between the simulated joint moments.

studies, i.e. Skinner et al. (2015), our findings suggested great variability and differences in the knee moment as well, as a natural consequence of the energy transferred from the ankle plantaflexors to the knee.

Our results depict the lower-limb motor alterations that are hallmark and primary manifestations of PD from a biomechanical perspective. Furthermore, we aimed at describing these motor symptoms in terms of motor control in order to understand how movement variables are controlled and coordinated (Mazzoni et al. 2012). We hypothesized that estimation of muscle forces could be a valuable tool to assess changes in the subject's strength over time, and might be proposed as metric to monitor the PD motor control deficiencies, given that the solely EMG is not descriptive of linear variations in strength, thus allowing a deeper understand in the cause-effect relationships between muscle co-activation and force distribution around a joint and how it is altered by PD. Studies assessing EMG signals during gait analysis revealed increased TA activity during the swing phase and a reduced amplitude of the signal, while a poor modulation in the leg extensor muscles during the stance phase was denoted (Albani et al. 2003). GL has shown a reduced amplitude of its activity during the stance phase in PD (Lewis et al. 2000). In terms of muscle forces little is known and there is no evidence 
A

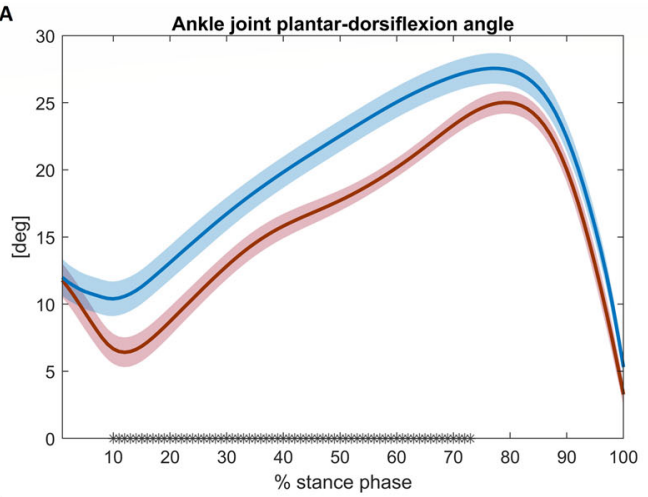

B
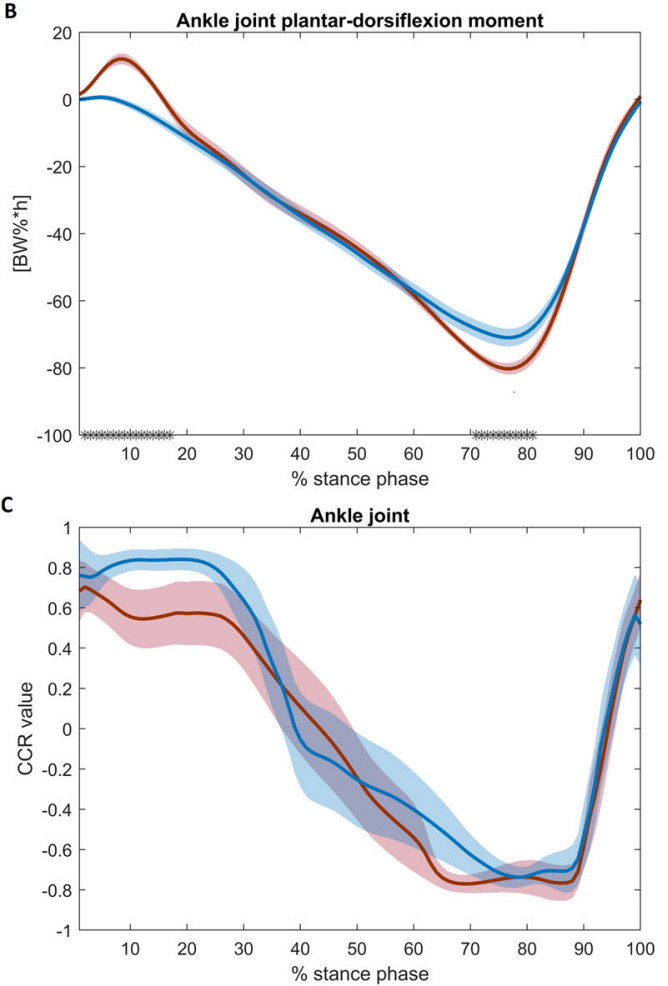

D

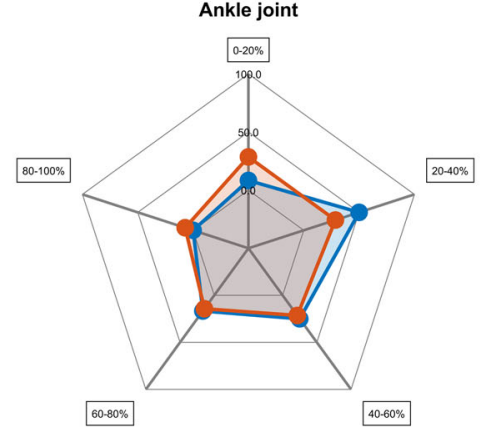

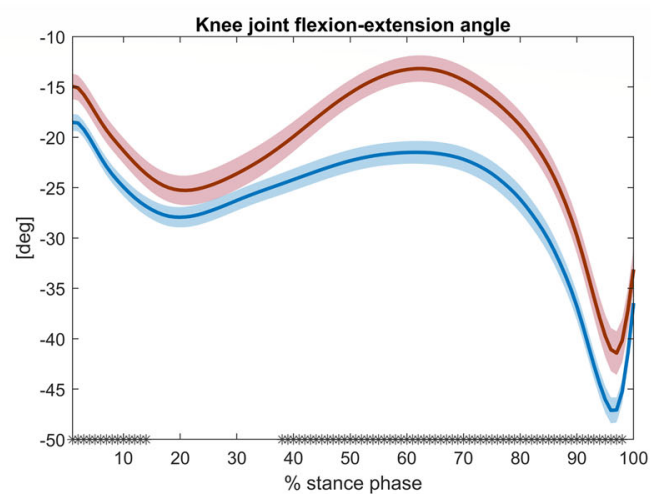

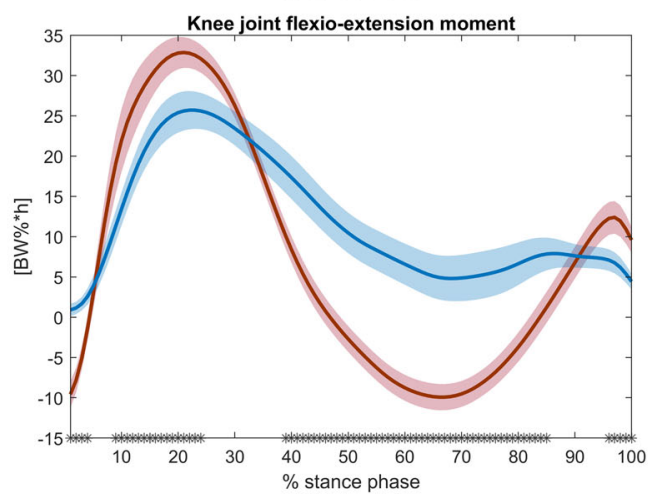

Knee joint

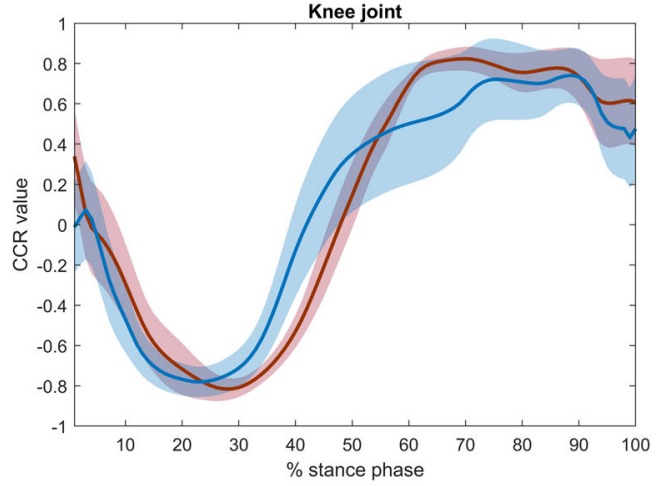

Knee joint

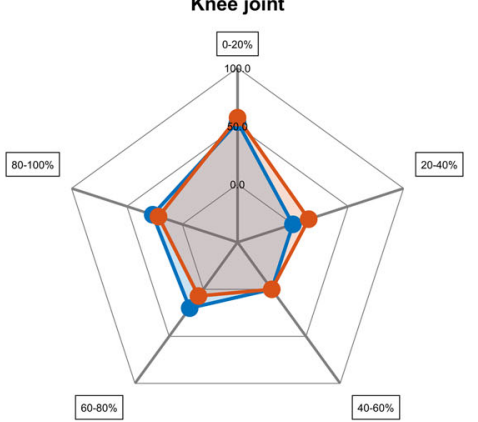

Figure 4. (A) Ankle and knee joint angles in percentage of the stance phase expressed in degrees, estimated via IK. CS (mean in red, \pm 1 standard error in shaded red area) in comparison with PD subjects (mean in blue, \pm 1 standard error in shaded blue area). (B) Ankle and knee joint moments in percentage of the stance phase and normalized with respect to body weight $\times$ height $(\mathrm{BW} \times \mathrm{h}) \%$, estimated via CEINMS. CS (mean in red, \pm 1 standard error in shaded red area) in comparison with PD subjects (mean in blue, \pm 1 standard error in shaded blue area). (C) CCR estimated from the activations generated via CEINMS. CS (mean in red, \pm 1 standard error in shaded red area) in comparison with PD subjects (mean in blue, \pm 1 standard error in shaded blue area). The ratio is presented in percentage of the stance phase and varies between -1 and 1 . (D) Co-contraction occurrence during five stages of the stance phase $(0-20 \%, 20-40 \%, 40-60 \%, 60-80 \%, 80-100 \%)$ for the ankle and knee joint. Data were normalized over the maximum occurrence value. CS (in red) in comparison with PD subjects (in blue). 

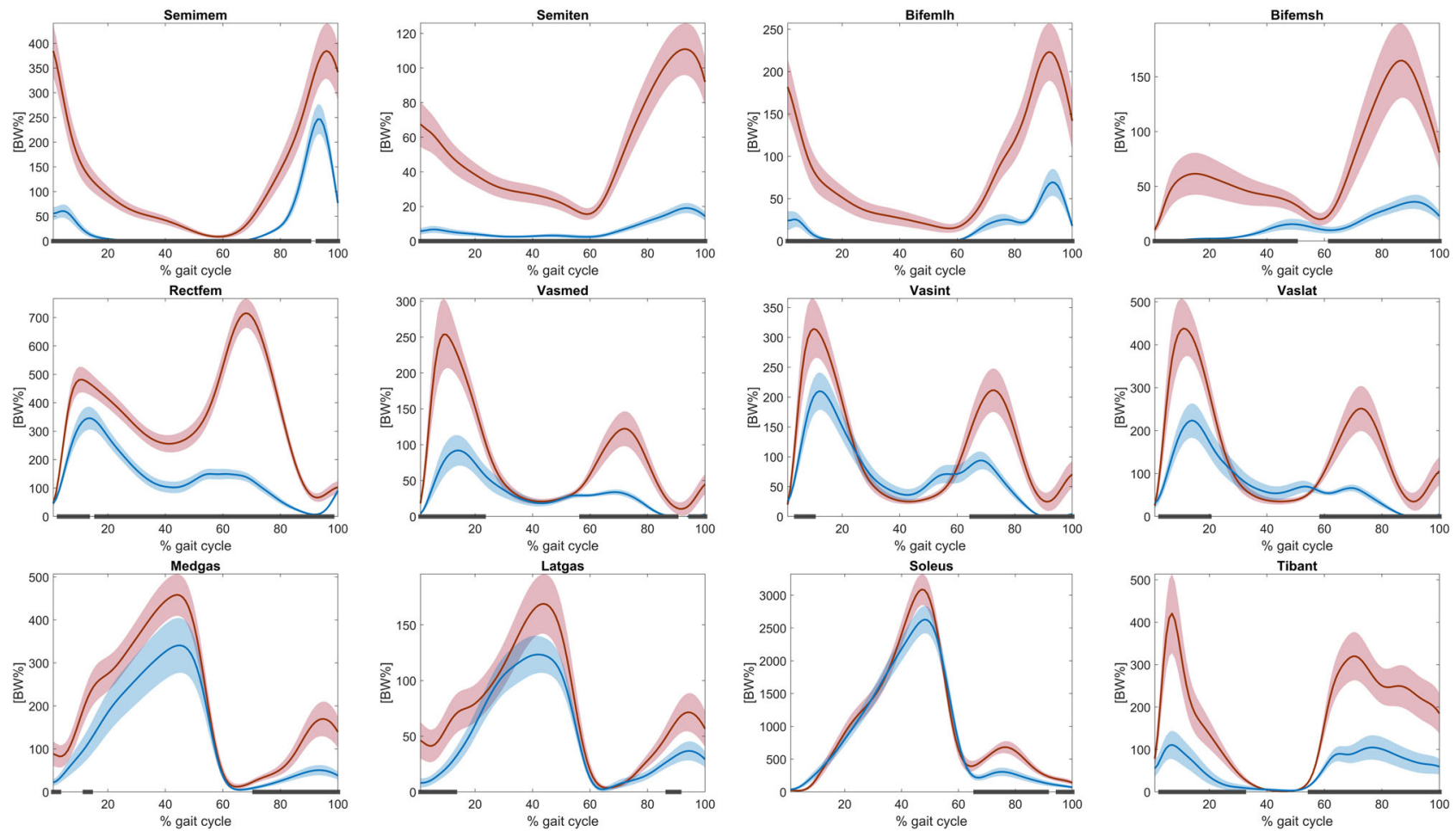

Figure 5. Muscle forces estimated with CEINMS. CS (in red, \pm 1 standard error in shaded red area) in comparison with PD subjects (in blue, \pm 1 standard error in shaded blue area). The muscle forces are presented in percentage of the gait cycle and normalized with respect to body weight (BW)\%. $\mathbf{\square}=\mathrm{p}<0.05$.

that this might be confirmed. To this end, this study investigates a method to estimate internal motion variables, for the first time, in people with PD in a clinical environment, with an approach that both considers their motor dysfunctions and neurological impairments, providing promising basis for the definition of advanced and more quantitative biomarkers. Therefore, the limitations derived from the musculoskeletal modeling approaches that employ optimization techniques to estimate muscle activations that do not account for impaired motor control in PD, have been overtaken, by the adoption of an EMG-informed modeling technique, thus allowing a more physiological estimation of muscle forces, as the results reported in Figure 2 showed. The results showed that MTU in PD patients produced significantly less force and displayed a higher amount of force variability than the CS, as reported in Stemach et al (Stelmach et al. 1989). Prior studies (Inkster et al. 2003) with similar findings suggested that the potential mechanism for a deficit in muscle force production and variability may be related to irregularities of single motor units in PD, attributed to an alteration of central input to the motor neuron pool and an abnormal drive from the basal ganglia to the thalamus. Literature reviews reported a consistently weaker force production in PD persons concerning the ankle joint
(Skinner et al. 2019), as confirmed by the results reported in Figure 5, where statistically significant difference in force magnitude were observed, mainly in knee joint flexors. Although the GL reduced amplitude activity reported in the previous quoted work was confirmed by a reduction in force in our results, the altered activity of TA was not consistent with the produced force, as it was found to be consistently lower $(0.0001<\mathrm{p}<0.0357)$ for PD both in the initial stance and swing phase.

The results of our work should provide an additional information to clinicians that could be used to plan intervention treatments aiming to restore and improve muscle forces. The model proposed herein provides a quantitative and repeatable metric to define the motor capacity of the patient, reducing the possible sources of variability affecting the clinical scales within and between clinician raters (Heldman et al. 2011). Moreover, the model provides information that could not be measured experimentally, including muscle force, which are directly related to movement capacity and can be used to track movement improvement or deterioration in a direct way. Thus, allowing the design of more effective rehabilitation treatments personalized on the subject functional capabilities. Muscle forces and co-contraction indexes might represent a compact representation of 
neuromuscular progress in between therapy evaluations. Furthermore, tracking the disease progression with the proposed method would be a helpful feedback for the physicians in both adjusting the dosage and the target of the physical intervention. Moreover, this approach will allow to comprehend how assistive and wearable technologies should interact with the user in order to provide an appropriate support and to restore a more physiological neuromusculoskeletal functions profile, estimating the degree of muscle forces required by the specific subject.

Must be said that the study comes with several limitations and, thus, should be acknowledged. Firstly, the reduced number of EMG recorded have impacted on the quantitative information on the internal variables, which still lack of a gold standard available for their estimation, yet it has been assessed and quantified (Table 2). However, future work will be focused on recording more EMGs in $\mathrm{PD}$, considering the use of HD-EMG and decomposition methods to capture activity of single motor units in patients, thereby obtaining higher fidelity neuro-mechanical processes. Nevertheless, it should be mentioned that the proposed technique allows overcoming some limitations of standard musculoskeletal modeling techniques (i.e. OpenSim static optimization (Delp et al. 2007)) which purely relies on optimization, tuned on non-altered neural pathways, by the adoption of a data-driven method, i.e. EMG-informed musculoskeletal modeling. This allows us to capture realistic patterns of PD population's muscle activation by means of EMGs and using them in a forward dynamics approach to virtually model the movements produced by both the skeletal and muscular system as controlled by the neural system (Buchanan et al. 2004). Secondly, the EMG envelope normalization was not performed based on MVC values. Measuring MVCs on patients was not possible as it would have made the test too lengthy for the patients. Therefore, a WTN was adopted, as previously performed in Varotto et al. (2017). The loss of information between a model calibrated with the MVCTN and WTN has been assessed and quantified (Table 2), showing promising results in the use of WTNr model for clinics. However, besides limitations, the setup we adopted in this work produced results that have proven to be consistent both at EMG and dynamic levels (Figures 2 and 3), allowing a quantitative analysis of differences in muscle forces and co-activation strategies between the CS and the PD participants. Thirdly, we did not include in our analysis muscle related to the action of hip joint, which plays a primary role in gait strategies together with the ankle joint. However, in future studies, the multi DOF model of Pizzolato et al. (2015) could be adopted and muscle controlling hip joint could provide additional understanding in the magnitude of force production and in PD's motor control. Lastly, the small sample size: however, it was possible to detect statistically significant changes in almost all the assessed variables, even though, the number of the processed subjects was poor.

Further studies are needed to confirm these preliminary results and, as clinical practice urges to assess the participants without the burden of instrumentation, future efforts need to focus on the definition of an optimal experimental setup to drive the model.

\section{Acknowledgments}

Marco Romanato wishes to acknowledge Fondazione Fresco Parkinson Institute Italia Onlus for the financial contribution to his PhD course. The part of this work performed by Annamaria Guiotto, was supported by MIUR (Italian Minister for Education) under the initiative 'Departments of Excellence' (Law 232/2016).

\section{Disclosure statement}

The authors declare no conflict of interest.

\section{References}

Albani G, Sandrini G, Künig G, Martin-Soelch C, Mauro A, Pignatti R, Pacchetti C, Dietz V, Leenders KL. 2003. Differences in the EMG pattern of leg muscle activation during locomotion in Parkinson's disease. Funct Neurol. 18(3):165-170.

Blanc Y, Dimanico U. 2010. Electrode placement in surface electromyography (sEMG) "Minimal Crosstalk Area" (MCA). TOREHJ. 3(1):110-126.

Buchanan TS, Lloyd DG, Manal K, Besier TF. 2004. Neuromusculoskeletal modeling: estimation of muscle forces and joint moments and movements from measurements of neural command. J Appl Biomech. 20(4): 367-395.

Busse ME, Wiles CE, van Deursen RWM. 2005. Muscle coactivation in neurological conditions. Phys Therapy Rev. 10(4):247-253.

Cappozzo A, Catani F, Della Croce U, Leardini A. 1995. Position and orientation in space of bones during movement: anatomical frame definition and determination. Clin Biomech (Bristol, Avon). 10(4):171-178.

Caramia C, Torricelli D, Schmid M, Munoz-Gonzalez A, Gonzalez-Varga J, Grandas F, Pons JL. 2018. IMU-based classification of Parkinson's disease from gait: a sensitivity analysis on sensor location and feature selection. IEEE J Biomed Health Inform. 22(6):1765-1774. 
Daliri MR. 2013. Chi-square distance kernel of the gaits for the diagnosis of Parkinson's disease. Biomed Signal Process Control. 8(1):66-70.

Davico G, Pizzolato C, Lloyd DG, Obst SJ, Walsh HPJ, Carty CP. 2020. Increasing level of neuromusculoskeletal model personalisation to investigate joint contact forces in cerebral palsy: a twin case study. Clin Biomech (Bristol, Avon). 72:141-149.

de Lau LML, Breteler MMB. 2006. Epidemiology of Parkinson's disease. Lancet Neurol. 5(6):525-535.

Del Din S, Carraro E, Sawacha Z, Guiotto A, Bonaldo L, Masiero S, Cobelli C. 2011. Impaired gait in ankylosing spondylitis. Med Biol Eng Comput. 49(7):801-809.

Delp SL, Anderson FC, Arnold AS, Loan P, Habib A, John CT, Guendelman E, Thelen DG. 2007. OpenSim: opensource software to create and analyze dynamic simulations of movement. IEEE Trans Biomed Eng. 54(11): 1940-1950.

Delp SL, Loan JP, Hoy MG, Zajac FE, Topp EL, Rosen JM. 1990. An interactive graphics-based model of the lower extremity to study orthopaedic surgical procedures. IEEE Trans Biomed Eng. 37(8):757-767.

Dorsey ER, Bloem BR. 2018. The Parkinson pandemic-a call to action. JAMA Neurol. 75(1):9-10.

Durandau G, Farina D, Asin-Prieto G, Dimbwadyo-Terrer I, Lerma-Lara S, Pons JL, Moreno JC, Sartori M. 2019. Voluntary control of wearable robotic exoskeletons by patients with paresis via neuromechanical modeling. J Neuroeng Rehabil. 16(1):91.

Folstein FE, Folstein SE, McHugh PR. 1975. Mini-mental state. A practical method for grading the cognitive state of patients for the clinician. J Psychiatr Res. 12(3): 189-198.

Heldman DA, Giuffrida JP, Chen R, Payne M, Mazzella F, Duker AP, Sahay A, Kim SJ, Revilla FJ, Espay AJ. 2011. The modified bradykinesia rating scale for Parkinson's disease: reliability and comparison with kinematic measures. Mov Disord. 26(10):1859-1863.

Hicks JL, Uchida TK, Seth A, Rajagopal A, Delp SL. 2015. Is my model good enough? Best practices for verification and validation of musculoskeletal models and simulations of movement. J Biomech Eng. 137(2):020905.

Hoehn MM, Yahr MD. 1967. Parkinsonism: onset, progression and mortality. Neurology. 17(5):427-442.

Inkster LM, Eng JJ, MacIntyre DL, Stoessl AJ. 2003. Leg muscle strength is reduced in Parkinson's disease and relates to the ability to rise from a chair. Mov Disord. 18(2):157-162.

Lewis GN, Byblow WD, Walt SE. 2000. Stride length regulation in Parkinson's disease: the use of extrinsic, visual cues. Brain. 123(10):2077-2090.

Mantoan A, Ceseracciu E, Spolaor F, Sawacha Z, Reggiani M. 2015. On the effect of EMG normalization in muscle forces estimation when using a multi-DOF EMG-driven neuromusculoskeletal model. V International Symposium on Computer Simulation in Biomechanics; July 9-11; Edinburgh, UK.

Mantoan A, Pizzolato C, Sartori M, Sawacha Z, Cobelli C, Reggiani M. 2015. MOtoNMS: a MATLAB toolbox to process motion data for neuromusculoskeletal modeling and simulation. Source Code Biol Med. 10:12.
Marek K, Jennings D, Lasch S, Siderowf A, Tanner C, Simuni T, Coffey C, Kieburtz K, Flagg E, Chowdhury S, et al. 2011. The Parkinson progression marker initiative (PPMI). Prog Neurobiol. 95(4):629-635.

Mazzoni P, Shabbott B, Cortes JC. 2012. Motor control abnormalities in Parkinson's disease. Cold Spring Harb Perspect Med. 2(6):a009282.

Morris ME, Iansek R, McGinley J, Matyas T, Huxham F. 2005. Three-dimensional gait biomechanics in Parkinson's disease: evidence for a centrally mediated amplitude regulation disorder. Mov Disord. 20(1):40-50.

Movement Disorder Society Task Force on Rating Scales for Parkinson's Disease. 2003. Mov Disord. 18(7): 738-750.

Peppe A, Paravati S, Baldassarre MG, Bakdounes L, Spolaor F, Guiotto A, Pavan D, Sawacha Z, Bottino S, Clerici D, et al. 2019. Proprioceptive focal stimulation (Equistasi®) may improve the quality of gait in middle-moderate Parkinson's disease patients. Double-blind, doubledummy, randomized, crossover, Italian multicentric study. Front Neurol. 10:998.

Pistacchi M, Gioulis M, Sanson F, De Giovanni E. 2017. Gait analysis and clinical correlations in early Parkinson's disease. FN. 32(1):28-34.

Pizzolato C, Lloyd DG, Sartori M, Ceseracciu E, Besier TF, Fregly BJ, Reggiani M. 2015. CEINMS: a toolbox to investigate the influence of different neural control solutions on the prediction of muscle excitation and joint moments during dynamic motor tasks. J Biomech. 48(14):3929-3936.

Rastegari E, Azizian S, Hesham A. 2019. Machine learning and similarity network approaches to support automatic classification of Parkinson's diseases using accelerometerbased gait analysis. Proceedings of the 52nd Hawaii International Conference on System Sciences, Hawaii.

Ricciardi C, Amboni M, De Santis C, Improta G, Volpe G, Iuppariello L, Ricciardelli G, D’Addio G, Vitale C, Barone P, et al. 2019. Using gait analysis' parameters to classify Parkinsonism: a data mining approach. Comput Methods Programs Biomed. 180:105033.

Romanato M, Sartori M, Durandau G, Volpe D, Sawacha Z. 2019. An EMG-informed modelling approach for the prediction of internal variables during locomotion in Parkinson's disease patients: a feasibility study. Gait Posture. 74:32-33.

Sartori M, Farina D, Lloyd DG. 2014. Hybrid neuromusculoskeletal modeling to best track joint moments using a balance between muscle excitations derived from electromyograms and optimization. J Biomech. 47(15): 3613-3621.

Sartori M, Fernandez JW, Modenese L, Carty CP, Barber LA. 2017. Toward modeling locomotion using electromyography-informed 3D models: application to cerebral palsy. WIREs Syst. Biol. Med. 9(2):e1368.

Sartori M, Llyod DG, Farina D. 2016. Neural data-driven musculoskeletal modeling for personalized neurorehabilitation technologies. IEEE Trans Biomed Eng. 63(5): 879-893.

Sartori M, Maculan M, Pizzolato C, Reggiani M, Farina D. 2015. Modeling and simulating the neuromuscular mechanisms regulating ankle and knee joint stiffness during human locomotion. J Neurophysiol. 114(4):2509-2527. 
Sartori M, Reggiani M, Farina D, Lloyd DG. 2012. EMGdriven forward-dynamic estimation of muscle force and joint moment about multiple degrees of freedom in the human lower extremity. PLoS One. 7(12):e52618.

Sawacha Z, Guarneri G, Avogaro A, Cobelli C. 2010. A new classification of diabetic gait pattern based on cluster analysis of biomechanical data. J Diabetes Sci Technol. 4(5):1127-1138.

Scarton A, Jonkers I, Guiotto A, Spolaor F, Guarneri G, Avogaro A, Cobelli C, Sawacha Z. 2017. Comparison of lower limb muscle strength between diabetic neuropathic and healthy subjects using OpenSim. Gait Posture. 58: 194-200.

Shetty S, Rao YS. 2016. SVM based machine learning approach to identify Parkinson's disease using gait analysis. 2016 International Conference on Inventive Computation Technologies (ICICT); Coimbatore, India. p. $1-5$.

Skinner JW, Christou EA, Hass CJ. 2019. Lower extremity muscle strength and force variability in persons with Parkinson disease. J Neurol Phys Ther. 43(1):56-62.

Skinner JW, Lee HK, Roemmich RT, Amano S, Hass CJ. 2015. Execution of activities of daily living in persons with Parkinson disease. Med Sci Sports Exerc. 47(9): 1906-1912.

Sofuwa O, Nieuwboer A, Desloovere K, Willems A-M, Chavret F, Jonkers I. 2005. Quantitative gait analysis in Parkinson's disease: comparison with a healthy control group. Arch Phys Med Rehabil. 86(5):1007-1013.
Spolaor F, Volpe D, Pavan D, Guiotto A, Fichera F, Torresin P, Fantinato E, Sawacha Z. 2017. Surface EMG analysis in Parkinson's disease patients before and after underwater gait training. Gait Posture. 57:53-54.

Stelmach GE, Teasdale N, Phillips J, Worringham CJ. 1989. Force production characteristics in Parkinson's disease. Exp Brain Res. 76(1):165-172.

Tiple D, Fabbrini G, Colosimo C, Ottaviani D, Camerota F, Defazio G, Berardelli A. 2009. Camptocormia in Parkinson disease: an epidemiological and clinical study. J Neurol Neurosurg Psychiatry. 80(2):145-148.

Varotto C, Sawacha Z, Gizzi L, Farina D, Sartori M. 2017. Closed-loop EMG-informed model-based analysis of human musculoskeletal mechanics on rough terrains. IEEE Int Conf Rehabil Robot. 2017:364-368.

Veerkamp K, Schallig W, Harlaar J, Pizzolato C, Carty CP, Lloyd DG, van der Krogt MM. 2019. The effects of electromyography-assisted modelling in estimating musculotendon forces during gait in children with cerebral palsy. J Biomech. 92:45-53.

Volpe D, Pavan D, Morris M, Guiotto A, Iansek R, Fortuna S, Frazzitta G, Sawacha Z. 2017. Underwater gait analysis in Parkinson's disease. Gait Posture. 52:87-94.

Volpe D, Spolaor F, Sawacha Z, Guiotto A, Pavan D, Bakdounes L, Urbani V, Frazzitta G, Iansek R. 2020. Muscular activation changes in lower limbs after underwater gait training in Parkinson's disease: a surface EMG pilot study. Gait Posture. 80:185-191. 